\title{
PAPER
}

Cite this: Phys. Chem. Chem. Phys., 2013, 15, 19213

Received 12th July 2013,

Accepted 19th September 2013

DOI: $10.1039 / c 3 c p 52919 c$

www.rsc.org/pccp

\section{Electronic excited states of a strongly correlated organic radical 1,3,5-trithia-2,4,6-triazapentalenyl (TTTA) adsorbed on a Si(001) surface}

\author{
K. Shudo, ${ }^{\star a c}$ T. Satake, ${ }^{\text {b }}$ T. Shimatsu, ${ }^{\text {b }}$ M. Uchiyamac and J. Takeda ${ }^{a}$
}

Electronic excited states of a strongly correlated organic radical, 1,3,5-trithia-2,4,6-triazapentalenyl (TTTA), adsorbed on a $\mathrm{Si}(001)$ surface were investigated by means of two-photon photoemission spectroscopy (2PPE) to elucidate the functional organic thin-film formation on a typical semiconductor substrate. The spectra were interpreted with the aid of density functional theoretical calculations. The unpaired electron of TTTA forms a covalent bond with the dangling bond of the Si-dimer initially, and there are resonant states of TTTA to Si near the surface. The molecules adsorbed at room temperature form dimers having diamagnetic properties at thicknesses of a few monolayers, while the paramagnetic phase appears at multilayer thickness. From the change in the work function, the orientation of the adsorbed TTTA molecules was determined to change depending on the thickness of the adsorbed layer.

\section{Introduction}

Since organic molecules have discrete electronic levels that can be designed by synthesis, the films of functional organic materials are considered to have a potential future utility in a wide variety of applications, ${ }^{1}$ in contrast to conventional inorganic crystalline and amorphous films. Beyond switching devices based on metal-organic-semiconductor structures, ${ }^{2}$ photovoltage, photoconductivity and photochromism have been investigated extensively in recent years. ${ }^{3,4}$ In particular, the application of bistable organic materials, whose two phases can be controlled by external electric or magnetic stimulation, is also expected for future devices, if their controllability is retained when they are fabricated on solid surfaces. In the present study, we focus on 1,3,5-trithia-2,4,6triazapentalenyl (TTTA), which exhibits photo-induced phase transition (PIPT). ${ }^{5-7}$

TTTA is a promising candidate for various applications, because its crystal shows photoreactive and magnetic-fieldresponsive properties between diamagnetic low temperature (LT) and paramagnetic high temperature (HT) phases at room temperature (RT). ${ }^{8}$ These properties are ascribed to its strongly correlated electron system, originating from an unpaired electron at the nitrogen terminal (Fig. 1). When TTTA forms a molecular crystal, the unpaired electrons interact and a drastic change is

\footnotetext{
${ }^{a}$ Faculty of Engineering/Science, Yokohama National University, Tokiwadai 79-5, Hodogaya-ku, Yokohama 240-8501, Japan.E-mail: ken1@ynu.ac.jp

${ }^{b}$ Graduate School of Engineering, Yokohama National University, Tokiwadai 79-5, Hodogaya-ku, Yokohama 240-8501, Japan

${ }^{c}$ Riken (The Institute of Physical and Chemical Research), Hirosawa 2-1, Wako-shi, Saitama 351-0198, Japan
}

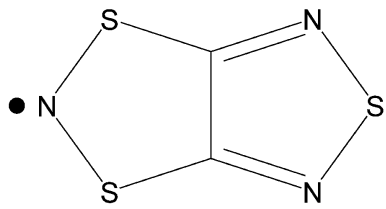

Fig. 1 The structure of a TTTA molecule. The black dot represents an unpaired electron at the nitrogen atom.

observed in the magnetic properties between the LT and HT phases. The HT phase is a Mott insulator owing to the strong correlation of unpaired electrons, and dimerization occurs to form the diamagnetic LT phase when the crystal is cooled. TTTA crystals have large hysteresis in the room temperature region owing to their magnetic bistability, ${ }^{8,9}$ and show PIPT from the LT to HT phase inside the hysteresis loop. ${ }^{10,11}$ When the LT phase is irradiated with intense light pulses, a nonlinear process can be observed; a photo-generated self-trapped exciton state with local lattice distortion transforms into the macroscopic photo-induced HT phase.

The stability of the films is generally affected by the substrate, and electronic states near the Fermi level may differ from bulk crystals because bond-formation in terms of moleculesubstrate interaction at the interface would be different from that of molecule-molecule interaction in crystalline structures. This point is essential for the application of bistable materials with these interesting properties. Nonetheless, no experimental results have been reported to show the electronic states clearly. Particularly, for use on semiconducting materials that easily form covalent bonds, the influence of the substrate is inevitable. ${ }^{12}$ 
Thus, electronic states, molecular orientation and bond formation must be clarified because the bistable states are very sensitive to slight changes in the electronic states near the Fermi level.

Two-photon photoemission (2PPE) spectroscopy can provide information on the electron in normally empty (excited) states in a conduction band as well as those in normally occupied (ground) states in a valence band. Similar to conventional ultraviolet photoelectron spectroscopy (UPS), probe pulses of UV light cause photoemission of electrons in the ground state through two-photon absorption of the pump pulse light. Contrary to inverse photoemission spectroscopy (IPES), which is also capable of probing normally empty states using relatively high energy electrons, 2PPE spectroscopy, as is used in this study, is superior for non-destructive identification of unoccupied states associated with photoexcitation. The information on excited and ground states is simultaneously obtained using ePPE spectroscopy, indicating that the electronic phototransitions can also be elucidated. This aspect is critical in differentiating electronic characteristics associated with the electronic correlation near the Fermi level, because they are not simply shifted from those in the isolated molecules. We address these issues by conventional UPS and 2PPE during the organic TTTA films growth, where their molecular orientation and related electronic states may change. Interpretation of the results is aided by electronic energies calculated using the standard theoretical methods.

\section{Experimental method}

TTTA was synthesized following a previously reported method. ${ }^{13}$ Single crystals of TTTA, grown by vacuum sublimation, were flakes with a typical size of $0.3-0.8 \mathrm{~mm}$ diameter and $0.2 \mathrm{~mm}$ thickness. These were placed in a glass test tube, which was then sealed and evacuated. The vapor pressure of TTTA is so high that TTTA gas from the tube, maintained at $30-35{ }^{\circ} \mathrm{C}$, could be introduced through a precise leak valve into an ultrahigh vacuum chamber, where the spectra were obtained.

We used a strip of an n-type single crystal $\mathrm{Si}(001)$ wafer (P-doped, 1.0-10.0 $\Omega \mathrm{cm}, 8.0 \times 6.0 \times 0.6 \mathrm{~mm}^{3}$ ) as a substrate. This was mounted on a molybdenum head that can be cooled to liquid nitrogen temperature, and placed in the vacuum chamber with a base pressure below $7.0 \times 10^{-8} \mathrm{~Pa}$. A clean surface of this Si substrate was prepared by repeated cycles of Ar-ion sputtering and flashing at $1150{ }^{\circ} \mathrm{C}$ several times, followed by slow cooling to $\sim 700{ }^{\circ} \mathrm{C}$. The third harmonic of a regenerative Ti:sapphire laser (central wavelength: $800 \mathrm{~nm}$, repetition rate: $1 \mathrm{kHz}$, pulse duration: $150 \mathrm{fs}, h \nu=4.65 \mathrm{eV}$ ) was used as an excitation light source for 2PPE. The beam was weakly focused on the surface at a spot with a diameter smaller than $0.1 \mathrm{~mm}$. The photoelectron energy was measured using a hemispherical energy analyzer (Vacuum Generator: CLAM-2). The electrons were detected normal to the surface, corresponding to the $\Gamma$ point in the reciprocal space. The sample was exposed to TTTA gas introduced into the vacuum chamber at $\sim 10^{-7} \mathrm{~Pa}$, and the exposure was expressed in Langmuir $\left(=1.0 \times 10^{-6}\right.$ Torr $\left.\mathrm{s}\right)$.
We measured the electronic spectra of sample films at the following steps. First, TTTA was sublimed to form a thin film on the substrate at RT. Next, the TTTA-saturated sample was cooled and allowed to transform into the LT phase. Finally, we measured the spectrum of the adsorbed TTTA film at low temperature $(200 \mathrm{~K})$ and again as the temperature was raised.

\section{Results}

\section{PPE spectra}

A typical set of 2PPE spectra is shown in Fig. 2 for various amounts of TTTA exposure on a clean $\mathrm{Si}(001)$ surface at RT. The horizontal axis shows the intermediate energy measured from the Fermi level $\left(E_{\mathrm{F}}\right)$, obtained after subtraction of the photon energy $(h \nu)$ from the final kinetic energy $\left(E_{\mathrm{K}}\right)$, and the vertical axis shows the photoemission intensity. To aid in the assignment of the energy of the initial state in the case of two-photon absorption, the initial energy $\left(E_{\mathrm{K}}-2 h \nu\right)$ is scaled at the top. The intensity at the energy of the lower edge (corresponding to the work function $\phi$ ) is high due to scattered electrons, and the edge changes according to the amount of exposure as indicated by arrows. $\phi$ was $5.1 \mathrm{eV}$ on the clean surface, then declined at exposure levels of 0.5-1.0 L, and increased when the exposure was more than $1.0 \mathrm{~L}$. In the following discussion, we ignore the apparent peak from the scattered components near the lower edge, because it originates after the decay process and is not directly related to the electronic states specific to Si and TTTA.

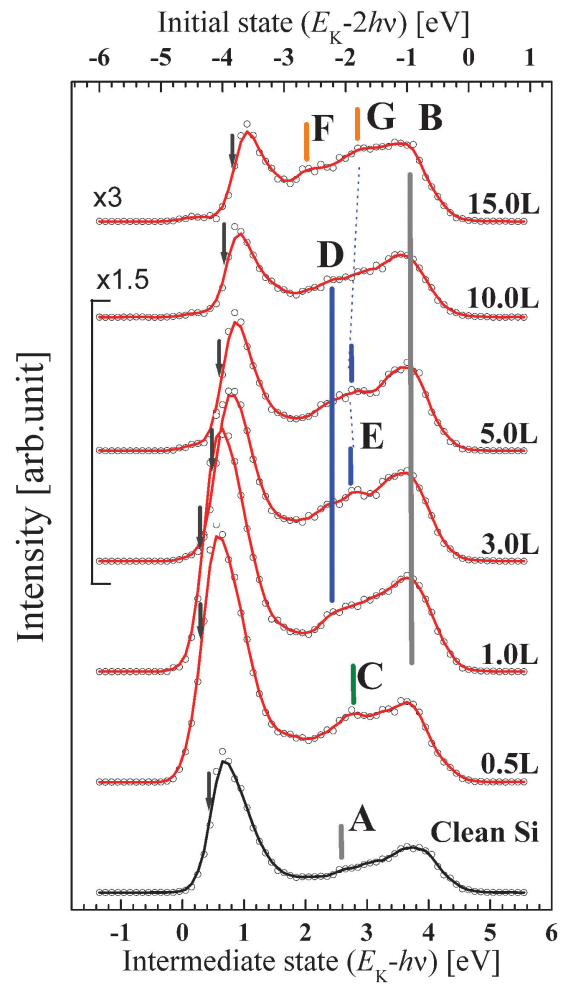

Fig. 2 2PPE spectra of TTTA/Si(001) at TTTA exposures of 0-15 L. The spectra were taken using $150 \mathrm{fs}$ pulses at $h \nu=4.65 \mathrm{eV}(267 \mathrm{~nm})$. Arrows indicates lower cut-off energy, corresponding to $\phi-h \nu$. 


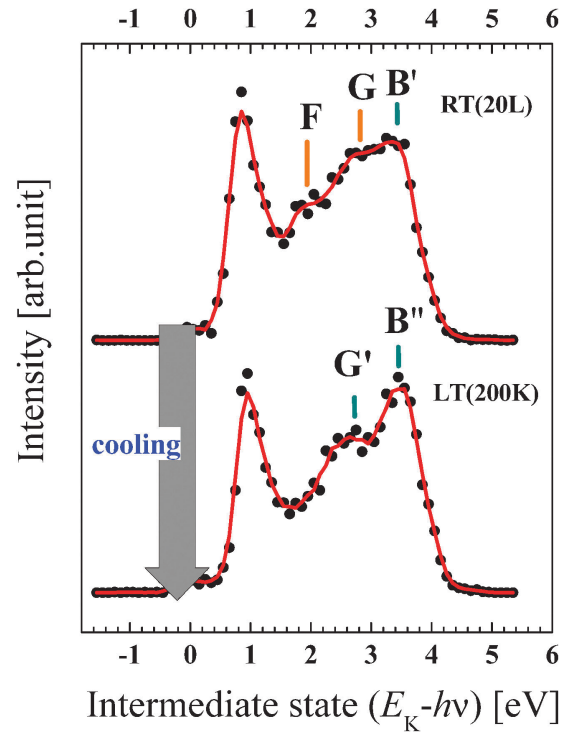

Fig. 3 2PPES spectral change of cooled TTTA/Si(001) adsorbed $(20 \mathrm{~L})$ at room temperature (RT) and cooled to lower temperature (LT: $200 \mathrm{~K}$ ).

In Fig. 2, the spectrum obtained from the clean $\mathrm{Si}(001)$ surface was in good agreement with a previous report on the $2 \times 1$ Si-dimer structure surface. ${ }^{14}$ On the clean surface, two electronic states were observed: peaks A and B at 2.6 and 3.7$3.8 \mathrm{eV}$, respectively. The latter, assigned to the bulk state of the crystalline Si substrate, ${ }^{14}$ remains present throughout. After 0.5 L TTTA exposure, a component was observed at $2.7 \mathrm{eV}$ (peak C), which is assigned to the electronic state of TTTA initially adsorbed on the Si surface. When the exposure reached $3 \mathrm{~L}$ and $5 \mathrm{~L}$, peaks $\mathrm{D}$ and $\mathrm{E}$ were formed successively. After the exposure reached $15 \mathrm{~L}$, new electronic states appeared at $\sim 2.0 \mathrm{eV}$ (peak F) and at $\sim 2.8 \mathrm{eV}$ (peak G). As the HT and LT phases are both stable in the RT region, as mentioned above, these two phases may both be relevant to the observed spectra.

At the exposure of $20.0 \mathrm{~L}$ or more, peaks $\mathrm{F}$ and $\mathrm{G}$ remained (shown in Fig. 3). This indicates that the surface covered with TTTA has a saturated structure. When the saturated sample was cooled to low temperature (approximately $200 \mathrm{~K}$ ), peak G shifted slightly (denoted as $\mathrm{G}^{\prime}$ ), whereas peak F (near $\sim 2 \mathrm{eV}$ ) disappeared. The broad peak B shifted to $3.5 \mathrm{eV}$ (peak $\mathrm{B}^{\prime}$ ) at high exposure, and become very sharp at $3.4 \mathrm{eV}$ (peak $\mathrm{B}^{\prime \prime}$ ) after cooling.

When TTTA was adsorbed onto the Si surface at low temperatures ( $200 \mathrm{~K}$ or below), reduction of the work function was much larger, suggesting that thicker film can be formed than at RT. No clear peak was found in 2PPE spectra from the TTTA adsorbed at the low temperatures, contrary to that adsorbed at RT shown in Fig. 3. However, with the temperature increasing, peak $\mathrm{G}^{\prime}$ appeared weakly at $\sim 257 \mathrm{~K}$, and peaks $\mathrm{F}$ and $\mathrm{G}$ emerged at 278-289 K. From these results on TTTA adsorbed at the low temperatures, we conclude that the thin film formed at low temperatures is crystallized in the LT phase at $\sim 257 \mathrm{~K}$, and that the phase transition from the LT to the HT phase takes place thermally at $278-289 \mathrm{~K}$ in such thin TTTA films formed on $\mathrm{Si}$ at low temperatures. The difference in the transition

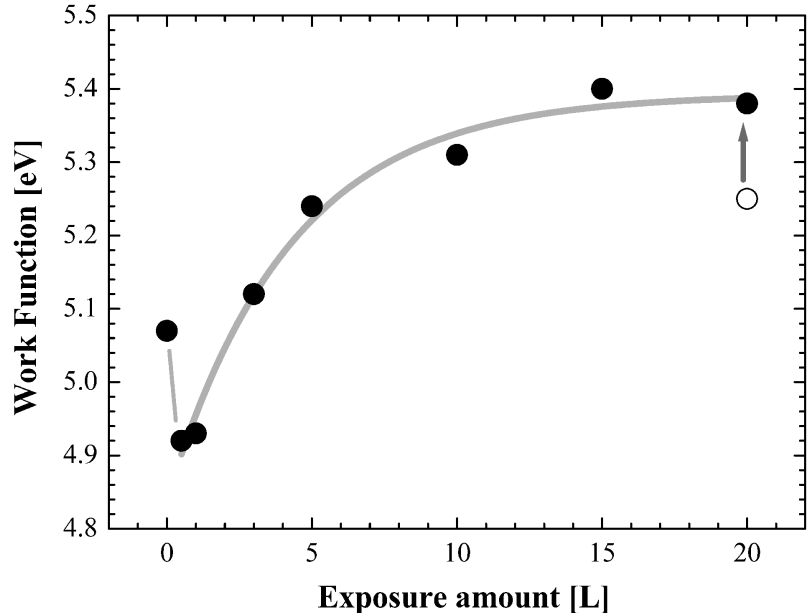

Fig. 4 Observed change of the work function $\phi$ of Si(001) with TTTA adsorbed at $6.5 \times 10^{-7} \mathrm{~Pa}$ at RT. The horizontal axis shows the amount of TTTA in terms of gas exposure. Data (closed circles) are taken from the series of measurements in Fig. 2. In the case of $20 \mathrm{~L}, \phi$ after adsorption at RT (open circle) increased to $\sim 5.4 \mathrm{eV}$ after cooling; see the text for details.

temperature between the solid-state TTTA previously reported $(\sim 305 \mathrm{~K})$ and our thin films $(\sim 280 \mathrm{~K})$ might come from the effect of the substrate; the phase transition in thin films is more affected by heating due to the photo-irradiation than the solid-state TTTA.

Fig. 4 shows the change of the work function $\phi$ as a function of the TTTA exposure amount at RT. The work function of clean $\operatorname{Si}(001)$ was $\phi=5.1 \mathrm{eV}$. At the initial stage of adsorption, the work function decreased rapidly. At $0.5 \mathrm{~L}$ exposure, $\phi$ was decreased by $\sim 0.15 \mathrm{eV}$. After further adsorption, the work function increased and became higher than that of the clean surface. When the amount of exposure was increased to several $\mathrm{L}$, the work function increased to $\phi=5.1-5.3 \mathrm{eV}$. From 10 to $20 \mathrm{~L}$, most of the surface had $\phi$ values in the range $5.3-5.4 \mathrm{eV}$ (filled circles), though some regions had slightly lower values. This suggests that the multilayer film is slightly inhomogeneous in the RT region, possibly due to slight variations in the surface temperature. The trace of the major structure (filled circles) decays exponentially to $\sim 5.3 \mathrm{eV}$ after the dip at $\sim 1 \mathrm{~L}$. The surface was partly inhomogeneous in the higher exposure region (15-20 L), and the increase in $\phi$ stopped at a lower value than $5.3 \mathrm{eV}$ (open circle in Fig. 4). However, $\phi$ reached $\sim 5.3 \mathrm{eV}$ after the sample was cooled to $200 \mathrm{~K}$, and the fluctuation of $\phi$ was much smaller for the cooled sample.

\section{Calculations}

Electronic state and band calculations were done using the molecular orbital (MO) method, based on density functional theory (DFT), in order to interpret the experimental spectra. Electronic states of the isolated TTTA molecule were obtained using Gaussian09 in the B3LYP model with the $6-31 \mathrm{G}+(\mathrm{d})$ basis set. ${ }^{15}$ Eigenenergies of a single isolated molecule (monomer) and a dimer were also calculated. In the latter case, the intermolecular distance of the parallel-constrained bimolecule was obtained as $3.56 \AA$ after geometry optimization of TTTA-dimer 

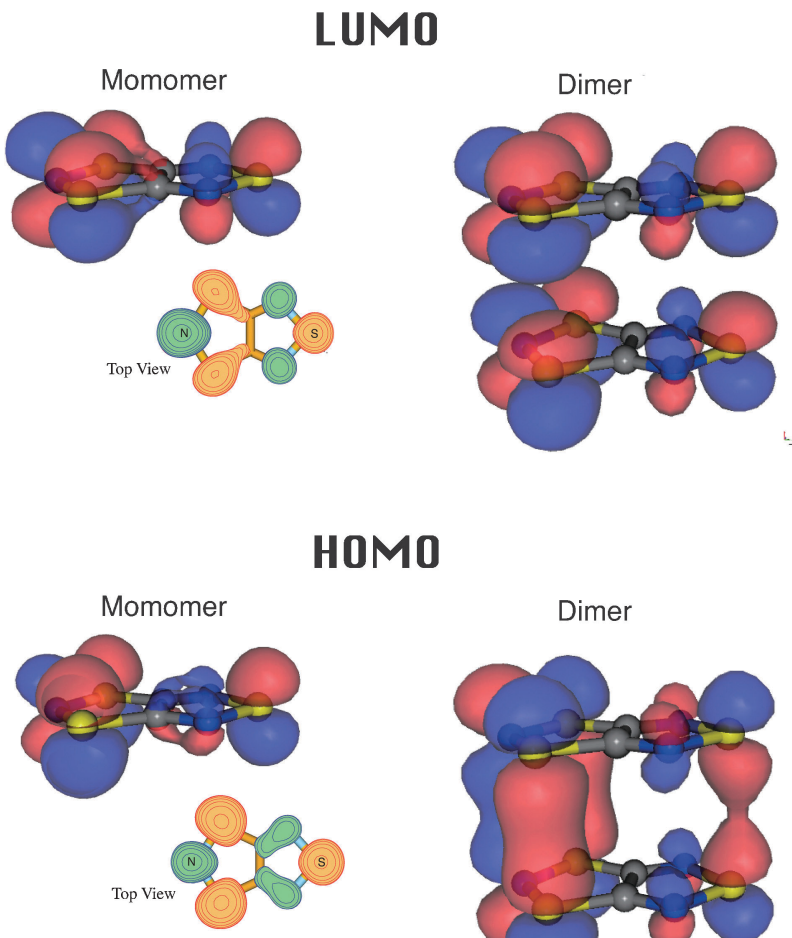

HOMO

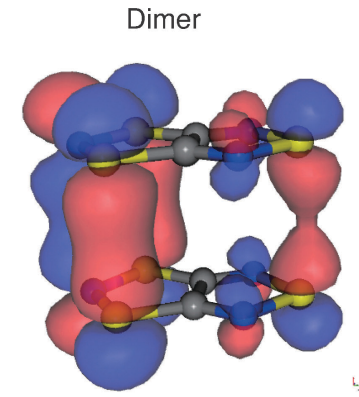

Fig. 5 Calculated structures, in terms of atomic positions, of an isolated single molecule and a dimer of TTTA. The distance between the two molecules in the dimer system is $3.56 \AA$. HOMO/LUMO orbitals are superimposed on the frameworks.

structure. Fig. 5 illustrates the wavefunction of the highest occupied molecular orbital (HOMO) and the lowest unoccupied molecular orbital (LUMO) of the monomer and the dimer. Each wavefunction has lobes perpendicular to the molecular plane (that is, a node), suggesting that it consists mainly of p-orbitals of each atom perpendicular to the plane. Each HOMO/LUMO is distributed not only at the N-terminal, but also near both the $\mathrm{N}$ - and S-terminals. However, there is a nodal surface between $\mathrm{C}$ and $\mathrm{S}$ in the HOMO and between $\mathrm{C}$ and $\mathrm{N}$ in the LUMO in the monomer (refer to the top views). In the dimer, no nodal plane is formed between the two molecules in the HOMO due to the overlap of mirror-inverted molecular lobes. In the LUMO of the dimer system, there is a nodal plane between the two molecules. Only the sign of the molecular wavefunction is opposite between the HOMO and the LUMO. This LUMO instability supports the idea that the photoexcited states in the LT phase favor structural change. ${ }^{16,17}$ The crystals in the LT phase retain this dimer stabilization via the Yarn-Teller effect. Since the dimerization would not greatly perturb the electronic spectra, ${ }^{18}$ the electronic states calculated for the dimer structure may be close to those in the LT phase.

The calculated dipole moment and charge distribution of each atom, obtained from Mulliken analysis in Gaussian09, are listed in Table 1 (unit: electron). The dipole moment is directed from the $\mathrm{N}$ - to S-terminal in both the monomer and dimer. These values should be helpful for interpretation of changes in the work function.
Table 1 Charge distribution

\begin{tabular}{lrr}
\hline & Monomer & \multicolumn{1}{c}{ Dimer } \\
\hline S (terminal) & 0.5586 & 0.6497 \\
C & -0.2181 & -0.5456 \\
N (connected to C) & -0.3635 & -0.2094 \\
S (connected to C) & 0.5340 & 0.6851 \\
N (terminal) & -0.4636 & -0.5098 \\
Dipole [Debye] & 0.8127 & 2.067
\end{tabular}

In contrast to the dimerized LT phase, the HT phase takes a one-dimensional laminated structure and acts as a Mott insulator because of the strong intermolecular interaction originating from the unpaired electron. ${ }^{6}$ Thus, it should be taken into account that intermolecular coupling splits the band of the unpaired electrons when TTTA forms a crystal structure in the HT phase. In order to figure out the mid-gap states due to the unpaired outermost shell electrons with spin, an ultrasoft pseudo-potential can be introduced to represent the inner shells; the valence and conduction bands can be estimated at the same time. Theoretical calculations with this potential were performed using the first-principles molecular dynamics program STATE (Simulation Tool for Atom TEchnology), which can also provide the metallic state density. ${ }^{19}$ According to density functional theory (DFT), the augmented wavefunctions are expressed using a plane wave basis set, and a generalized gradient approximation (GGA) method was used. The structural parameters of the crystals, such as lattice constants, are taken from ref. 20. The cut-off energy of the wavefunctions was 20 Ry or higher, and the reciprocal space was divided into $6 \times 6 \times 6$ meshes for bulk crystal and $6 \times 6 \times 1$ meshes for surface slab. Fig. 6 shows the calculated electronic states of TTTA crystals in the LT and HT phases after structural optimization. The LT phase shows exactly the same curve for major and minor spins, but the HT phase has slightly different curves.

The calculated eigenenergies of the molecular monomer and dimer, obtained using Gaussian09, are also shown in Fig. 6, after a horizontal shift to fit $E_{\mathrm{F}}$ between the HOMO and the LUMO. Because the electrons in TTTA crystals have a very small dispersion even in the valence band, ${ }^{6} 8 \times 8 \times 8$ meshes or more yielded no effective difference in the state density with regard to resolution of the experimental spectra. The spectra plotted in Fig. 6 for both crystal phases are quite similar to each other, except for the states in the region between -3 to $+4 \mathrm{eV}$ from $E_{\mathrm{F} .}{ }^{20}$ This indicates that the electronic structure retains the character of isolated molecules, as there are peaks in the curves close to the molecular eigenenergies. This means that it is difficult to analyze electrons of TTTA with conventional UPS. ${ }^{21}$ In the HT phase, the unpaired electron splits into the bands at $\sim \pm 0.45 \mathrm{eV}$ from $E_{\mathrm{F}}$.

The unoccupied states of the crystal in the LT phase are distributed across $E_{\mathrm{F}}$, and crystallization affects the electrons in the region between approximately 0 and $\sim 3 \mathrm{eV}$ above $E_{\mathrm{F}}$. This suggests that the simple isolated dimer model is not adequate in this region, and that the correlation between dimers should be taken into account in the excited state region. Since 2PPE 


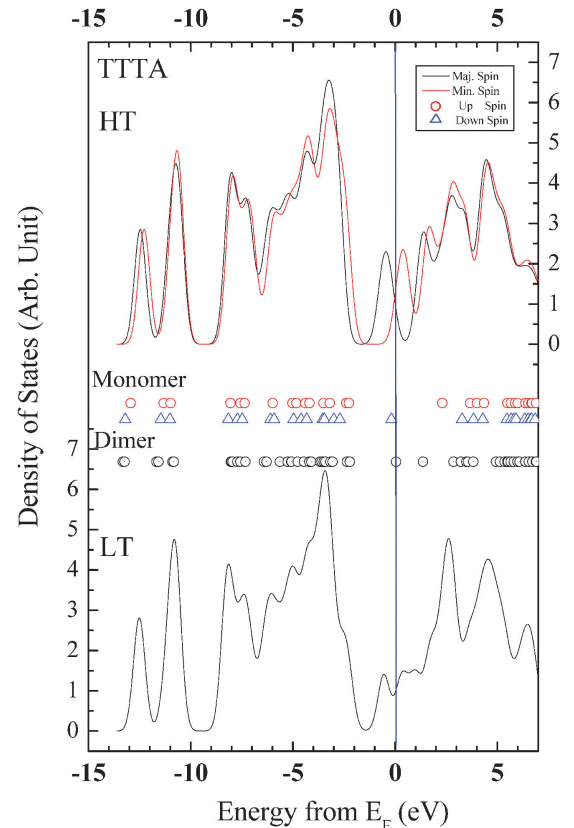

Fig. 6 Calculated electronic structure of TTTA. Curves show state densities of the HT and LT phases of TTTA crystals. The eigenenergy levels of the molecular monomer and dimer are plotted. Note that isolated monomer has major (triangle) and minor (circle) spins.

spectroscopy is capable of obtaining the spectra at energies above $E_{\mathrm{F}}$, it is a very powerful tool to analyze non-isolated systems of organic crystals.

\section{Discussion}

In this work, 2PPE spectroscopy was used to examine the changes of excited states generated by phototransition during absorption of increasing amounts of TTTA on a Si(001) surface, including structural phase transition. As the increase of TTTA exposure was accompanied by a decrease of emission intensity chiefly from Si (Fig. 2), the TTTA film thickness increased with increasing exposure of the surface to TTTA vapor. The decrease of the emission also suggests that photoemission from the excited states of TTTA chiefly involves electrons scattered from the substrate, rather than electrons derived from the ground states of TTTA. This idea is supported by the fact that there is no optical transition at the photon energy of $h \nu=4.65 \mathrm{eV}$ that we used, ${ }^{15}$ indicating that the efficiency of two-photon absorption inside the TTTA molecule would be low.

As shown in Fig. 2, peak B at $3.7 \mathrm{eV}$ is a state of the $\mathrm{Si}$ substrate (see spectrum of the clean Si surface). ${ }^{14}$ During adsorption, the position of peak $\mathrm{B}$ changes negligibly with regard to the work function change at $\sim 0.5 \mathrm{eV}$. This means no band bending near the surface when the surface is covered with adsorbate. Thus, charge transfer from/to the substrate is very small, in accordance with Fig. 6 , which shows that the band gap of TTTA is larger than that of Si except for the states involving the unpaired electron. Peak $\mathrm{C}$ at $2.76 \mathrm{eV}$ was observed only at the exposure of $0.5 \mathrm{~L}$ and presumably reflects initial

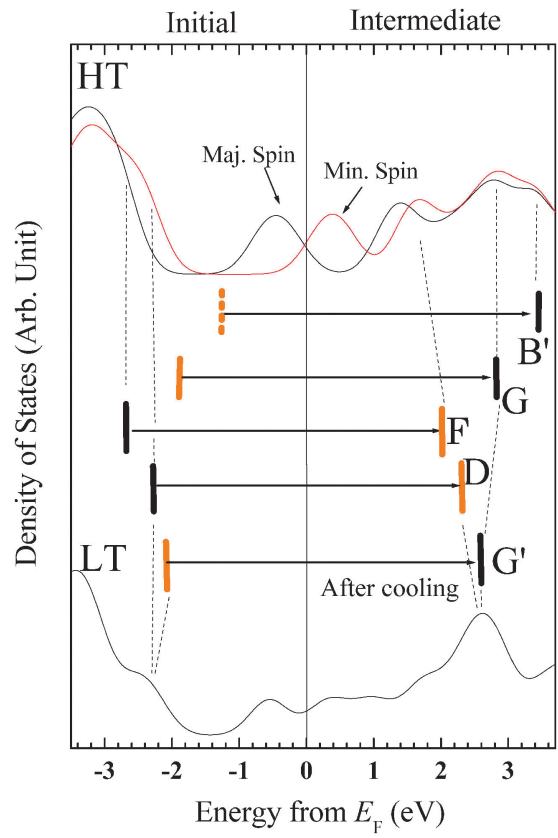

Fig. 7 Energies of the observed peaks. Possible initial and intermediate states corresponding to each peak are indicated by vertical bars. Solid bars indicate peaks assigned in the calculated electronic structure of TTTA (curves taken from Fig. 6), while orange bars are slightly discrepant. A dashed bar for $B^{\prime}$ has no state.

adsorption of TTTA. The interface structure of TTTA/Si(001) following initial adsorption will be discussed later, as the dangling bond of the Si surface in the substrate gap and the unpaired electron of TTTA may form new chemical bonds.

As the exposure was increased, the intensity of peak B becomes stronger in comparison with the electrons scattered from $\mathrm{Si}$ (spectral intensity at the edge of lower energy). Broadening (or slight shifting) of peak B at higher exposures might be associated with a state within TTTA resonant to the substrate. This TTTA state should be slightly lower than the Si state, because the film states (peaks $\mathrm{B}^{\prime \prime}$ and $\mathrm{B}^{\prime}$ ) appear sharper when the surface is covered by thicker films. Fig. 7 compares the calculated state density with the energy of peak $\mathrm{B}^{\prime}$ in Fig. 3 . There is a TTTA state at $E_{\mathrm{F}}+3.5 \mathrm{eV}$, which is weakly resonant to the Si state. However no TTTA states are found near the energy of the initial state $(-1.2 \mathrm{eV})$ in both phases. Thus, the state $\mathrm{B}^{\prime}$ is filled by scattering from Si states. We note that there are two branches, $X_{4}$ and $X_{1}$ from $\Gamma_{25^{\prime}}$, of the Si substrate in this region. ${ }^{14}$

In Fig. 7 , at $E_{\mathrm{F}}-2.2 \mathrm{eV}$, there is a weak peak in the LT phase and a shoulder in the minor spin curve for the HT phase, which originated from molecular eigenenergy near $E_{\mathrm{F}}-2.5 \mathrm{eV}$ (see Fig. 6). This is the position of the initial state of the observed. In the calculated state densities in the LT and HT phases, no clear peak is found at intermediate energy $(+2.2 \mathrm{eV})$, but there is a finite density of states. Thus, the peak originates from phototransition within the TTTA layer. In a conventional UPS experiment, there is a peak at $-2.4 \mathrm{eV}$ in the spectrum of TTTA on an Au surface. ${ }^{22}$ This ground state corresponds to the origin of peak $\mathrm{D}$, and two-photon absorption occurs through the finite 
density at $E_{\mathrm{F}}+2.2 \mathrm{eV}$. We cannot assign which phase the peak comes from. Disappearance of peak D from $10 \mathrm{~L}$ to $15 \mathrm{~L}$ suggests that the structure of the interface layer of TTTA film on the Si surface may be different from that of a thick film over few L. This peak is buried due to the emergence of peak $\mathrm{F}$ at $15 \mathrm{~L}$.

At the high exposure of $15 \mathrm{~L}$, peaks $\mathrm{F}$ and $\mathrm{G}$ are found at +2.0 and $+2.8 \mathrm{eV}$, respectively. Since peak $\mathrm{F}$ was observed only after high exposure, it presumably originates from thick crystal structure. From Fig. 7, we assign peak G to originate from a strong peak of the excited state in the HT phase that works as the intermediate state. At the energy corresponding to the intermediate and initial states of peak $\mathrm{F}$, there is no peak in the calculated state density. When we exposed a sample to $20 \mathrm{~L}$ at RT, then cooled it to $200 \mathrm{~K}$, which is below the phase transition temperature, peak $\mathrm{F}$ at $+2.0 \mathrm{eV}$ (seen at exposures of $15 \mathrm{~L}$ or more) disappeared. This observation indicates that the state originated from the HT phase. We conclude that the origin of peak $\mathrm{F}$ is the ground state of the crystal at $E_{\mathrm{F}}-2.7 \mathrm{eV}$, and photoemission occurs through peaks at 1.2-1.7 eV of the major and minor spins.

At the same time, peak $\mathrm{G}$ shifted to peak $\mathrm{G}^{\prime}$ at $0.2 \mathrm{eV}$ lower energy after cooling. This change can be explained in terms of phase transition from the HT to LT phase. The energy of peak $\mathrm{G}^{\prime}$ has a strong peak in the LT phase. Thus, peak E lies very close to peak $\mathrm{G}^{\prime}$, which originates from the crystalline LT phase. Since the film of the TTTA molecule at 3-5 L exposure consists of a few layers at most, it is difficult to cover the surface with films having dimerized structure. Dmerization at RT may occur partly under influence of the Si substrate when the film is very thin at low exposure.

Next, we go back to the topic of the work function (Fig. 4). Although the work function changes, we consider that the change is caused by the intrinsic polarity of the TTTA molecule. It should be noted that the molecule has a large dipole moment, and that the dipole per molecule is increased after dimerization (Table 1). In the initial adsorption phase, the dipole moment lies in the direction in which the work function decreases. Adsorption occurs with the unpaired electron downward to the Si surface. This situation is illustrated in Fig. 8(a). It can be seen that the unpaired electron easily forms a covalent bond with the dangling bond of the $\mathrm{Si}(001)$ surface, although this bond formation may reduce the dipole moment. During multilayer formation after further adsorption, the dipole moment aligns towards the direction in which the work function increases, so that the unpaired electron comes toward the vacuum, as illustrated in Fig. 8(b). The interfacial structure remains unclear, but some pairs of dimers must be located near the substrate surface (as mentioned above). In the temperature region where both phases are stable, dimers can be formed easily during rotation of the molecule. This is the reason why the work function scatters on the surface in multi-layer adsorption. Because the density of the dipole of the film is increased by the dimerization, the crystal orientation may exhibit a distribution, or dimer domains may be formed inhomogeneously in nanometer-scale film. Cooling the sample a) Initial adsorption (RT)

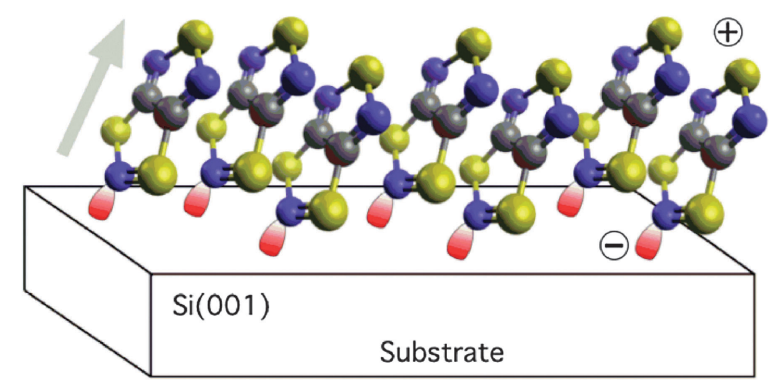

b) Multilayer adsorption (RT)

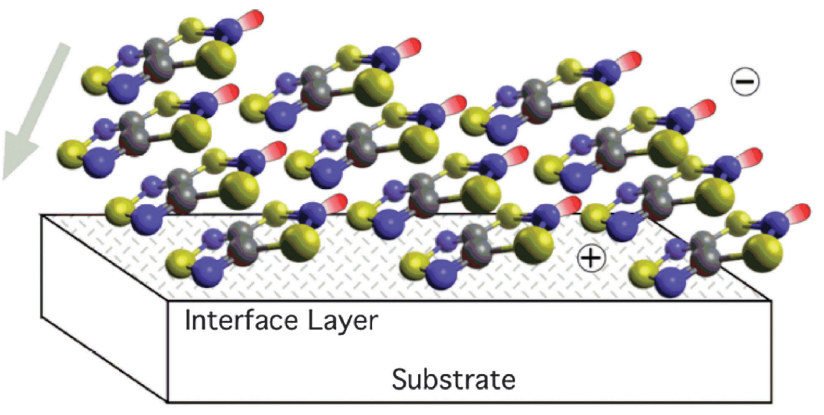

c) Dimerization (LT)

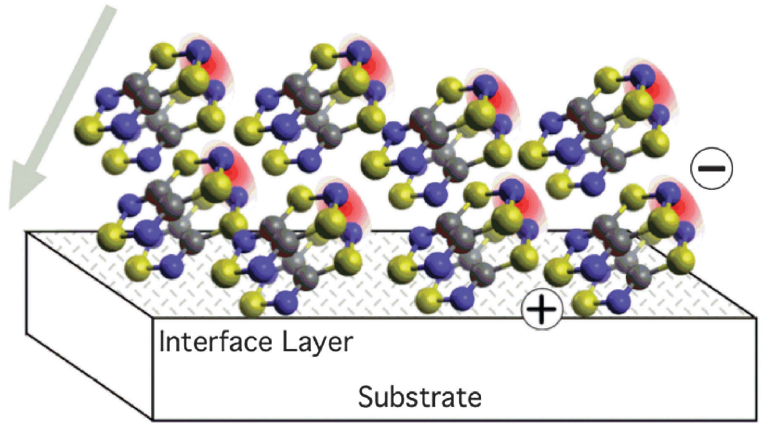

Fig. 8 Schematic structure of TTTA adsorbed on a Si(001) surface. At RT, (a) the $\mathrm{N}$-terminal is initially oriented to the surface up to monolayer coverage, (b) the S-terminal tends to be directed to the surface at multilayer thickness, and (c) after cooling to form the LT phase the molecules in the film are paired. Arrows indicate the dipole moment of the adsorbed layer.

forces the multilayer film to transform into the dimerized LT phase oriented to the vacuum at a stable angle, as illustrated in Fig. 8(c).

We move the topic to the adsorption process in order to estimate the work function and to elucidate peaks D and E yet unassigned clearly. We calculated model surfaces in two methods. One is $2 \times 2$ slabs of six Si layers calculated in GGA with STATE to obtain partial density of state in the TTTA molecule, and the other is clusters of surface slab containing a Si dimer $\left(\mathrm{Si}_{27} \mathrm{H}_{33}\right)$ for MO using Gaussian09 to obtain dipole moment through the electronic distribution by a natural bond orbital (NBO) and to reveal the origin of the states in terms of wavefunction. Both methods consistently gave similar stabilized structure. When TTTA approaches the surface, the N-terminal bonding to a dangling bond has lower energy by $\sim 1.9 \mathrm{eV}$ than 
(a)

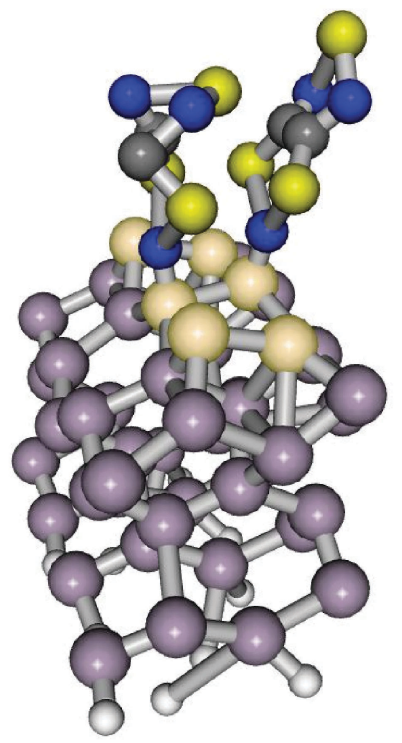

(b)

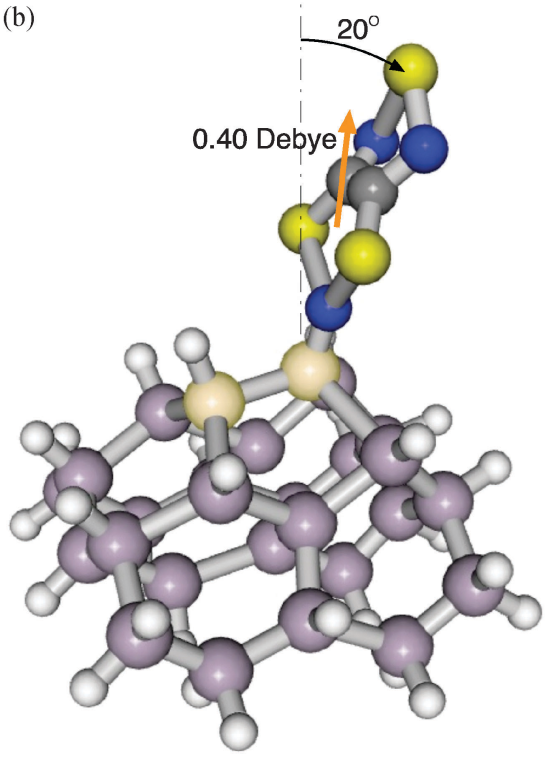

Fig. 9 (a) A stable structure of TTTA-adsorbed Si(001) slab of a $2 \times 2$-periodic supercell $(2 \times 3$ unit cells are shown). Blue and yellow spheres represent nitrogen and sulfur, respectively; purple spheres are Si except for light brown Si forming dimers. N-terminals bond to $\mathrm{Si}$ in the dimer. TTTA at down-Si (left) is much deformed than that at up-Si (right). (b) A single TTTA molecule adsorbed at an up-Si of a dimer in $\mathrm{Si}_{27} \mathrm{H}_{33}$ clusters. The TTTA molecule is inclined by $\sim 20$ degrees from the surface normal while the dipole moment of this molecule is inclined by a few degrees.

the S-terminal. This indicates that the $\mathrm{N}$-terminal bonding is preferably formed, as in Fig. 8(a). When the N-terminal of TTTA attacks a dangling bond of a down-Si in the dimer, the TTTA molecule is inclined and then it is deformed. When the $\mathrm{N}$-terminal approaches a dangling bond of an up-Si in the dimer, on the other hand, the TTTA molecule is stabilized if the dangling bond at the other side of the dimer is terminated by TTTA, a decomposed atom, or artificially introduced hydrogen. A typical stable structure is shown in Fig. 9(a). A stabilized TTTA on the up-Si is inclined by $20^{\circ}$ from the surface normal, as shown in Fig. 9(b). The TTTA molecule in this adsorbed

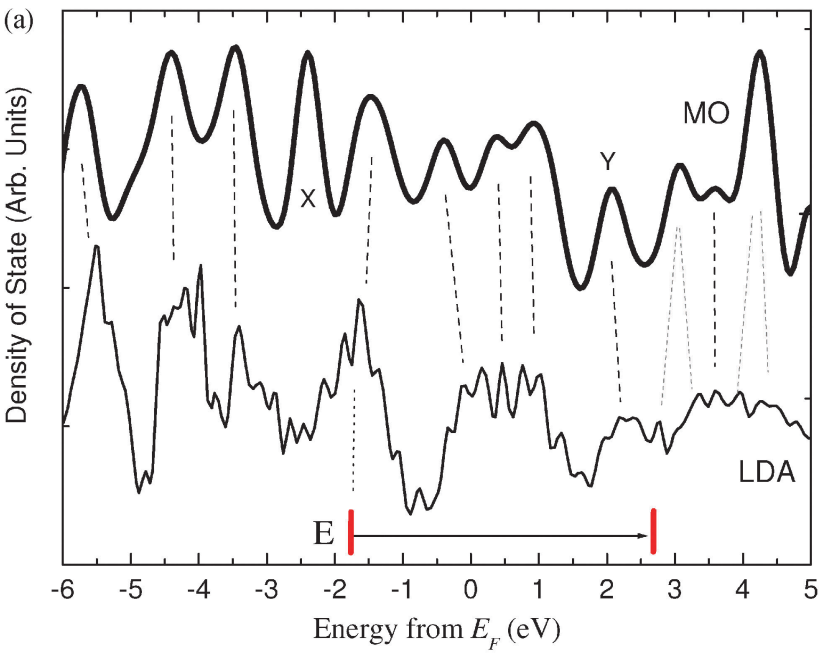

(b)

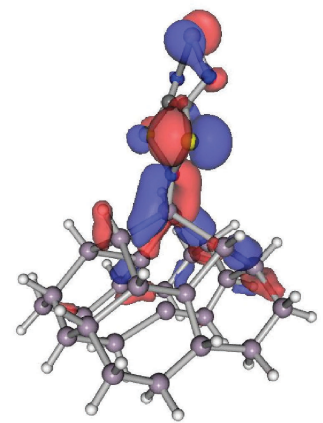

Fig. 10 (a) State densities of TTTA calculated with a cluster and a slab. Spectra of clusters with hydrogen at a down-Si and with/without the TTTA molecule at an up-Si of the dimer (Fig. 9(b)) are obtained from the calculated eigenvalues, and the difference of spectra by TTTA bonding is plotted (MO). Electrons in the $2 \times 2$ slab (structure in Fig. 9(a)) are projected to eight TTTA atoms, and the sum of the electronic state is plotted as a partial density of the molecular state (LDA). (b) A typical wave function at $E_{\mathrm{F}}-1.7 \mathrm{eV}$.

structure has 0.40 Debye of dipole moment nearly perpendicular to the surface, consistent with the picture obtained from the work function (Fig. 8(a)).

Peaks D and E, disappearing at the high exposures in Fig. 2, are related to the interfacial/adsorbed states between $\mathrm{Si}$ and TTTA. To assign the origins of the peaks, the density of state of the adsorbed molecule was calculated. Fig. 10(a) shows the calculated density of state, and the spectra from the two methods are very close to each other; corresponding peaks are connected using dashed lines. However, there is a discrepancy between the calculated two curves: a peak at $E_{\mathrm{F}}-2.4 \mathrm{eV}$ (marked with ' $\mathrm{X}$ ') lies only in the cluster, not in the projection of the molecular states in the slab. This comes from split and shift of the $\sigma$ bonding state between $\mathrm{Si}-\mathrm{Si}$ near the dimer ${ }^{23,24}$ due to the bond formation induced by TTTA adsorption. At the initial energy of the observed peak $\mathrm{E}(\sim-1.8 \mathrm{eV}$ in Fig. 2$)$, there is no calculated density of states in the crystalline phase (Fig. 7) while there is a peak in Fig. 10(a). Therefore, peak $\mathrm{E}$ is assigned to the local state of the adsorbed structure around $-1.7 \mathrm{eV}$. This state consists of resonant states among $\pi$ states of TTTA, a $\pi$ state of the Si-Si dimer, and back bonds of $\mathrm{Si}$ (an example is 
shown in Fig. 10(b)). As peak E disappears from the experimental spectra with the exposure increased, the photoemission process would be limited to the interface layer. Thus we conclude that peak corresponds to the phototransition from the TTTA-dimer resonant state to finite density in the adsorbed TTTA (found at 2.7-3.0 eV in the calculations). We next discuss the origin of peak D. Experimentally it originates from the intermediate state at $+2.4 \mathrm{eV}$ and the initial state at $-2.3 \mathrm{eV}$. There are two calculated peaks $\mathrm{X}$ and $\mathrm{Y}$ at the corresponding energy (peak X only in the MO curve). Thus, we assign that peak $D$ originates from the resonant phototransition between these states. Peak D appears prior to peak E, because a small amount of atoms decomposed from TTTA may contribute as well to the increase in this $\mathrm{Si}-\mathrm{Si}$ $\sigma$ bonding state by the shift/split of the surface local Si states. Finally, we note that peaks D and E emerge even in the very early stage of the film formation (estimated as the order of a monolayer adsorption), because these states come from the resonant phototransitions localized within the interface/adsorbed layer.

\section{Summary}

TTTA has potential as a material for organic-semiconductor application. We studied its adsorption on a $\mathrm{Si}(001)$ surface by means of 2PPE spectroscopy that is very sensitive near (especially above) $E_{\mathrm{F}}$. The ground states (below $E_{\mathrm{F}}$ ) of the crystalline phases retain the molecular character so strongly that conventional UPS is not applicable for evaluating the strongly correlated states, phase transition, and photoexitation processes. The interaction between molecules in the excited states, observed in the 2PPE spectra, revealed the formation of adsorbed states of the radical and structural phase transition, including interfacial states affected by bond formation. As an aid to interpret the spectra, DFT calculations were performed. At RT the molecules readily form dimers at the thickness of a few layers, while the HT phase appears at increased thickness (multilayer film). Orientation of the ad-molecules can be estimated from the change in the work function, as is consistent with the theoretical interpretation of the spectra in terms of adsorption process and interface states. The transition from the HT to LT phase resulted in changes in the spectral features. The electronic character above $E_{\mathrm{F}}$ in the range of a few $\mathrm{eV}$, which has an important influence on fabrication of organic-semiconductor systems and design of functional devices, is very sensitive to the adsorption amount and crystal formation in ultrathin films.

\section{Acknowledgements}

Computations were performed partly on the RIKEN Integrated Cluster of Clusters (RICC) and partly on supercomputer systems at the Institute for Solid State Physics (Univ. of Tokyo). K.S. thanks Prof. Y. Morikawa (Osaka Univ.) and Prof. K. Ohno (Yokohama Nat'l Univ.) for discussions on computational assistance and discussion on the calculation results. This work was supported by Grants-in-Aid for Scientific Research (Kakenhi; Grant Number 24655007, 22654034, 23241034, and 24655007) from Japan Society for the Promotion of Science and Ministry of Education, Culture, Sports, Science and Technology, Japan.

\section{References}

1 C. Martinelli, G. M. Farinola, V. Pinto and A. Cardone, Materials, 2013, 6, 1205-1236.

2 P. Sebastian, F. Lindner, K. Walzer, B. Lüssem and K. Leo, J. Appl. Phys., 2011, 110, 084508.

3 A. Tada, Y. Geng, Q. Wei, K. Hashimoto and K. Tajima, Nat. Mater., 2011, 10, 450-455.

4 S. Koshihara, Y. Tokura, K. Takeda and T. Koda, Phys. Rev. B: Condens. Matter Mater. Phys., 1995, 52, 6265-6272.

5 E. Collet, N. Moisan, C. Balde, R. Bertoni, E. Trzop, C. Laulhe, M. Lorenc, M. Servol, H. Cailleau, A. Tissot, M.-L. Boillot, T. Graber, R. Henning, P. Coppens and M. B.-L. Cointe, Phys. Chem. Chem. Phys., 2012, 14, 6192-6199.

6 K. Ohno, Y. Noguchi, T. Yokoi, S. Ishii, J. Takeda and M. Furuya, Eur. J. Chem. Phys. Chem., 2006, 7, 1820.

7 L. Radosinski, T. Luty, K. Nasu, H. Ohnishi, K. Nishioka, A. Radosz and D. Wójt, Acta Phys. Pol., A, 2012, 121, 359-368.

8 W. Fujita and K. Awaga, Science, 1999, 286, 261; W. Fujita and K. Awaga, Synth. Met., 2003, 137, 1263-1265.

9 J. Takeda, M. Imae, O. Hanado, S. Kurita, M. Furuya, K. Ohno and T. Kodaira, Chem. Phys. Lett., 2003, 378, 456-462.

10 T. Kon, S. Oguri, I. Katayama, T. Kodaira and J. Takeda, Phys. Rev. B: Condens. Matter Mater. Phys., 2009, 79, 035106.

11 I. Katayama, T. Kon, K. Mitarai and J. Takeda, Phys. Rev. B: Condens. Matter Mater. Phys., 2009, 80, 092103.

12 J. M. Buriak, Chem. Rev., 2002, 102, 1271-1308.

13 G. Wolmershuäuser and R. Johann, Angew. Chem., Int. Ed., 1989, 28, 920.

14 K. Shudo and T. Munakata, Phys. Rev. B: Condens. Matter Mater. Phys., 2001, 63, 125324.

15 http://www.gaussian.com.

16 I. Katayama, K. Mitarai, T. Kon and J. Takeda, J. Lumin., 2009, 129, 1931-1933.

17 S. Oguri, O. Hanado, J. Takeda, M. Furuya, K. Ohno, S. Inoue and T. Kodaira, J. Lumin., 2005, 129, 112, 283-286.

18 P. A. Pieniazek, S. E. Bradforth and A. I. Krylov, J. Chem. Phys., 2008, 129, 074104.

19 K. Toyoda, Y. Nakano, I. Hamada, K. Lee, S. Yanagisawa and Y. Morikawa, Surf. Sci., 2009, 603, 2912-2922.

20 M. Furuya, K. Ohno, T. Morisato, Y. Kawazoe and J. Takeda, Trans. Mater. Res. Soc. Jpn., 2003, 28, 911; M. Furuya, K. Ohno, J. Takeda and Y. Kawazoe, Trans. Mater. Res. Soc. Jpn., 2004, 29, 3719.

21 K. Iketaki, K. Kanai, W. Fujita, K. Awaga, J. Tsutsumi, H. Yoshida, N. Sato, M. Knupfer, Y. Ouchi and K. Seki, Chem. Phys. Lett., 2008, 451, 58-62; W. Fujita, A. Awaga, H. Matsuzaki and H. Okamoto, Phys. Rev. B: Condens. Matter Mater. Phys., 2002, 65, 064434.

22 K. Iketaki, K. Kanai, K. Ttsuboi, W. Fujita, K. Agawa, K. Knupfer, Y. Ouchi and K. Seki, Synth. Met., 2005, 153, 457-460.

23 G. V. Hansson and R. I. G. Uhrberg, Surf. Sci. Rep., 1988, 9, 197-292.

24 J. Ihm, M. L. Cohen and D. J. Chadi, Phys. Rev. B: Condens. Matter Mater. Phys., 1980, 21, 4592-4599. 